\title{
Directional velocity estimation using focusing along the flow direction - I: Theory and simulation
}

Jensen, Jørgen Arendt

Published in:

I E E E Transactions on Ultrasonics, Ferroelectrics and Frequency Control

Link to article, DOI:

10.1109/TUFFC.2003.1214505

Publication date:

2003

Document Version

Publisher's PDF, also known as Version of record

Link back to DTU Orbit

Citation (APA):

Jensen, J. A. (2003). Directional velocity estimation using focusing along the flow direction - I: Theory and simulation. I E E E Transactions on Ultrasonics, Ferroelectrics and Frequency Control, 50(7), 857-872. https://doi.org/10.1109/TUFFC.2003.1214505

\section{General rights}

Copyright and moral rights for the publications made accessible in the public portal are retained by the authors and/or other copyright owners and it is a condition of accessing publications that users recognise and abide by the legal requirements associated with these rights.

- Users may download and print one copy of any publication from the public portal for the purpose of private study or research.

- You may not further distribute the material or use it for any profit-making activity or commercial gain

- You may freely distribute the URL identifying the publication in the public portal 


\title{
Directional Velocity Estimation Using Focusing Along the Flow Direction I: Theory and Simulation
}

\author{
Jørgen A. Jensen, Senior Member, IEEE
}

\begin{abstract}
A new method for directional velocity estimation is presented. The method uses beamformation along the flow direction to generate data in which the correct velocity magnitude can be directly estimated from the shift in position of the received consecutive signals. The shift is found by cross-correlating the beamformed lines. The approach can find the velocity in any direction, including transverse to the traditionally emitted ultrasound beam. The velocity estimation is studied through extensive simulations using Field II. A 128-element, 7-MHz linear array is used. A parabolic velocity profile with a peak velocity of $0.5 \mathrm{~m} / \mathrm{s}$ is simulated for different beam-to-flow angles and for different emit foci. At $45^{\circ}$ the relative standard deviation over the profile is $1.6 \%$ for a transmit focus at $40 \mathrm{~mm}$. At $90^{\circ}$ the approach gave a relative standard deviation of $6.6 \%$ with a transmit focus of $80 \mathrm{~mm}$, when using 8 pulseecho lines and stationary echo canceling. Pulsatile flow in the femoral artery was also simulated using Womersley's flow model. A purely transverse flow profile could be obtained with a relative standard deviation of less than $10 \%$ over the whole cardiac cycle using 8 pulse emissions for each imaging direction, which is sufficient to show clinically relevant transverse color flow images.
\end{abstract}

\section{INTRODUCTION}

I $\mathrm{N}$ medical ultrasound, color flow mapping has been inItensively used for studying the instantaneous blood flow in the body. The blood vclocity is measured by emitting a series of ultrasound pulses in the same direction, and the velocity is then found by estimating the shift in position of the blood scatterers over time [1]. This can be done by either estimating a phase shift and relating this to the emitted frequency [2], or it can be found more directly as the shift in position of the scatterers through a crosscorrelation of consecutive received RF lines [3]. Both these techniques find the velocity component along the direction of the ultrasound beam. The projection of the velocity vector onto the ultrasound beam makes it impossible to detect a purely transverse flow, as no movement takes place along the beam. This is a major problem in the clinic, since most vessels run along the skin surfacc and, thus, perpendicular to the beam. Angling the transducer often presses on the vessel and alters the flow pattern. A full vector estimation of the velocity is, thus, of great diagnostic value.

Several authors have suggested methods for solving the

Manuscript received August 9, 2002; accepted January 28, 2003.

The author is with the Technical University of Denmark, Bldg. 348, DK-2800 Kgs. Lyngby, Denmark (e-mail: jaj@oersted.dtu.dk). transverse velocity estimation problem. Fox [4] suggested using two crossing beams from two active apertures. A triangulation scheme could then be used for finding both the axial and transverse flow. Using large transducers is often a drawback, and the standard deviation of the transverse components is dependent on the angle between the beams and, thus, on the depth in tissue. Trahey and coworkers [5] developed a speckle tracking approach, in which any velocity vector can be found. The approach uses a small part of the image as a tracking kernel on a larger search region. The method is applied on image data and puts a high demand on the fast acquisition of the data. The estimates also get uncertain, when the tracking kernel is small. Another idea is the introduction of a transverse oscillation in the ultrasound field [6], [7], where the motion transverse to the beam can be found. A similar approach has been described by Anderson [8]. Bonnefous [9] used the cross-correlation function on parallel beams to find the transverse component of the velocity vector. The approach unfortunately breaks down for a flow that is not transverse to the ultrasound beam. Jensen and Lacasa [10] suggested using received signals focused along the flow direction to solve this problem. Beams along the flow direction are then formed and used in the cross-correlation to find the velocity. The angle between the emitted beam and the flow direction has to be known or determined, but then the correct velocity magnitude can be found for any angle. An improvement of the method was presented by Jensen and Gori [11]. Here, a matched filter approach was used to improve the focusing of the flow beams, and this gave an improvement of nearly a factor of 5 on the standard deviation for a purely transverse flow. The price to pay was the large increase in number of calculations, because individual filters have to be applied on all elements of the receiving array.

This paper is the first of two that describes the directional beamforming and velocity estimation using a simplified focusing scheme. This first paper describes the theory behind the directional focusing approach and how it can be used for velocity estimation. A series of simulations are used for revealing the performance under realistic operating conditions. The accompanying paper [12] makes an experimental investigation of the approach using an experimental ultrasound research scanning system for revealing its performance for linear array flow imaging.

The method and delay calculations are described in Section II. A simulation model is introduced in Section III and 


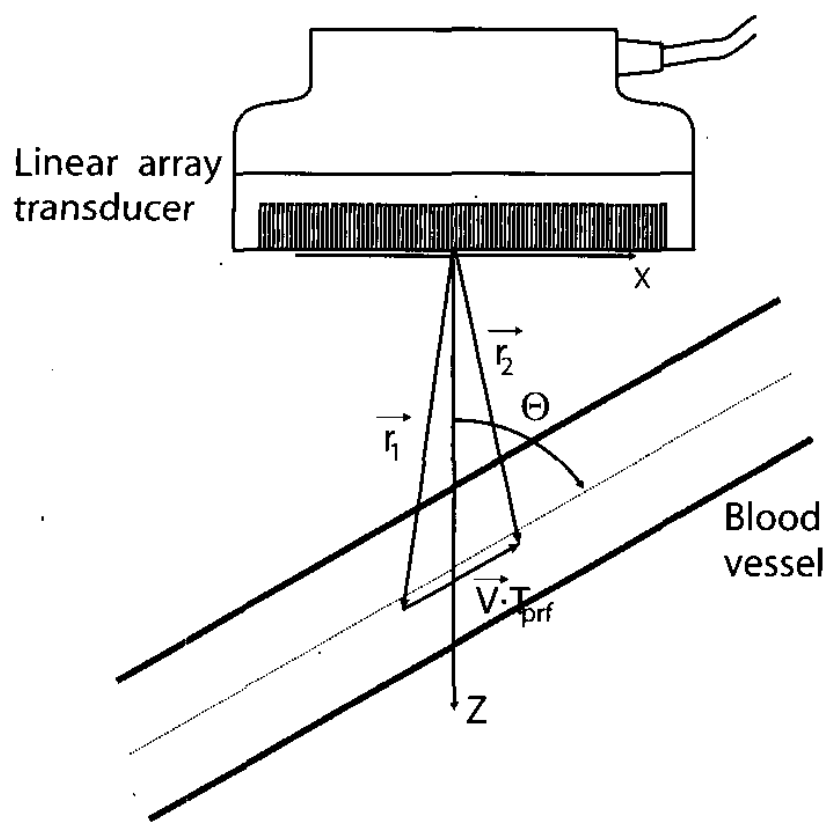

Fig. 1. Setup and coordinate system for velocity estimation with the ultrasound transducer at the top. The position of a single scatterer in the vessel is denoted with $\vec{r}_{1}$. It has moved to the position $\vec{r}_{2}$ after $T_{p r f}$ seconds.

comprehensive simulations are shown in Section IV which demonstrate that the approach is capable of accurately estimating the velocity vector. A single example of simulated transverse color flow mapping in the femoral artery is shown in Section V.

\section{Theory of Directional Velocity Estimation}

This section will decribe the new method for directional velocity estimation. First, the basic measurement situation is explained, and then the concept of directional signals are introduced, and it is shown how the velocity can be estimated from these: The measurements of such directional signals are derived, and finally some implementation details are given.

\section{A. Traditional Velocity Estimation}

The basic measurement setup is shown in Fig. 1. The transducer is shown on the top, and the flow is confined to the vessel below the transducer. The angle between the ultrasound beam and the vessel is $\theta$, where $90^{\circ}$ is a fully transverse flow. The coordinate system is given by $x, y, z$, where the $z$ coordinate is depth in tissue. It is assumed that the flow is laminar in the vessel and follows the vessel walls. The fluid consists of a number of particles, which radiate sound in all directions, when insonified by the ultrasound. The vector $\vec{r}_{L}$ indicates the location of such a scatterer. It has a velocity vector of $\vec{v}$, and after a time interval $T_{p r f}$, it has moved a distance $\vec{v} T_{\text {prf }}$ for an acceleration free flow, and its new location is denoted by $\vec{r}_{2}=\vec{r}_{1}+\vec{v} T_{p r f}$.

Current velocity estimation systems find the velocity along the ultrasound beam. This is done by emitting a focused ultrasound field in one direction and then beamform the received signal along the emitted beam. A second measurement is made in the same direction, and it is assumed that the beam is so wide, that the scatterers mainly stay within the ultrasound beam between measurements. The shift in position of the scatterers is translated into a time delay in the second signal given by [3], [13], and [14]

$$
t_{s}=\frac{2|\vec{v}| \cos (\theta)}{c} T_{p r f}
$$

where $c$ is the speed of sound. The time shift is estimated by cross-correlating the two received signals and then finding the peak position in the function. Hereby, only the velocity along the ultrasound beam direction is determined. The $\cos (\theta)$ dependence is a consequence of the beamformation being done in direction of the ultrasound propagation rather than in direction of the flow lines. The purpose of these papers is to demonstrate that beamformation can be done along the direction of the flow and that the correct velocity amplitude hereby can be found also for a purely transverse velocity direction.

\section{B. Basic Principle}

The basic method is to focus the received signals along the flow direction for a given depth. The signals for two emissions are then cross-correlated, and the shift between them is found. This is a shift in spatial position of the scatterers and dividing by the time between emissions, thus, directly gives the velocity magnitude. The angle between the emitted beam and the flow direction must be known before the beamformation can be done. The angle is assumed known in this paper and could, e.g., be found from the B-mode image as in conventional spectral velocity estimation.

The first step in the method is to introduce a coordinate system that is aligned along the flow rather than along the ultrasound beam direction. This is shown in Fig. 2. Here, the $x^{\prime}$-axis of the new coordinate system is parallel to the flow lines, and the center of the system is placed at the center of the vessel. The relation between the two coordinate systems is

$$
\begin{aligned}
& x=x^{\prime} \sin (\theta) \\
& y=y^{\prime} \\
& z=z^{\prime} \cos (\theta)+z_{\text {ves }},
\end{aligned}
$$

where $z_{\text {ves }}$ is the distance from the transducer surface to the center of the vessel. In the new coordinate system, the velocity is $\vec{v}=\left(v_{x^{\prime}}, 0,0\right)$, where $v_{x^{\prime}}=|\vec{v}|$. The position of the scatterer can now be expressed through a scalar, where the first position is $x_{1}^{\prime}$, and the position after $T_{p r f}$ seconds is $x_{2}^{\prime}=x_{1}^{\prime}+v_{x^{\prime}} T_{p r f}$. To find the velocity, a signal as a function of $x^{\prime}$ is obtained, where the first signal is given 


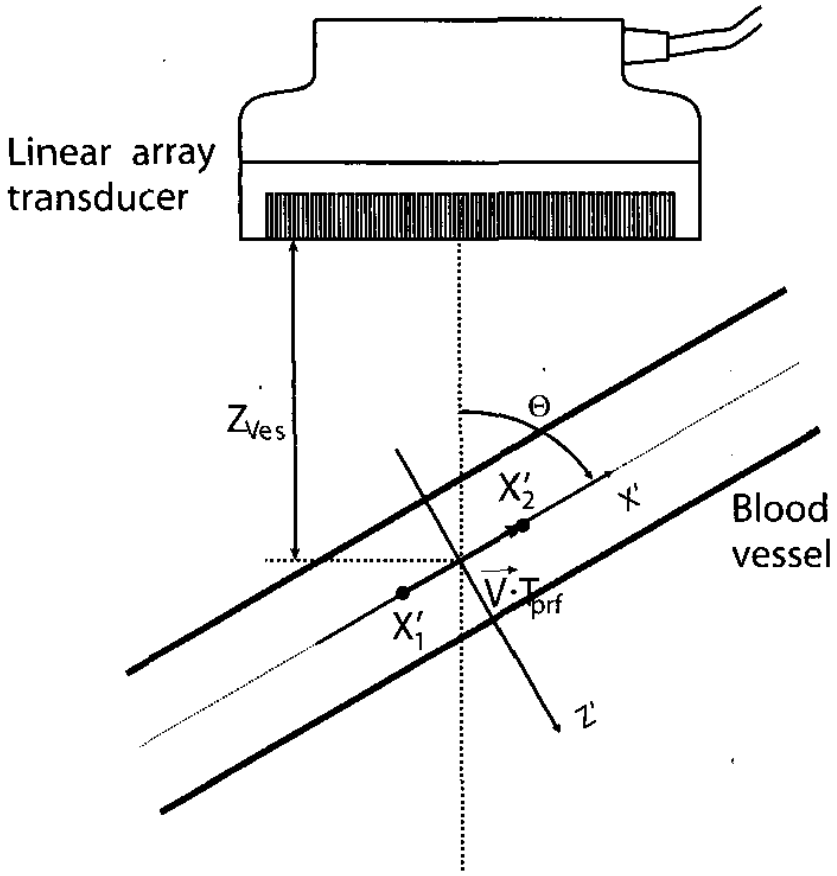

Fig. 2. Rotated and translated coordinate system oriented along the flow lines and with its center at the center of the vessel.

by $g_{1}\left(x^{\prime}\right)$, and the second obtained $T_{p r f}$ seconds later is $g_{2}\left(x^{\prime}\right)$. How to obtain these directional signals along the flow direction will be described in Section II-C.

The two signals are now related by

$$
g_{2}\left(x^{\prime}\right)=g_{1}\left(x^{\prime}-v_{x^{\prime}} T_{p r f}\right)
$$

Cross-correlating the two signals gives

$$
\begin{aligned}
R_{12}\left(\tau_{x^{\prime}}\right) & =\int_{X} g_{1}\left(x^{\prime}\right) g_{2}\left(x^{\prime}+\tau_{x^{\prime}}\right) d x^{\prime} \\
& =\int_{X} g_{1}\left(x^{\prime}\right) g_{1}\left(x^{\prime}+\tau_{x^{\prime}}-v_{x^{\prime}} T_{p r f}\right) d x^{\prime} \\
& =R_{11}\left(\tau_{x^{\prime}}-v_{x^{\prime}} T_{p r f}\right)
\end{aligned}
$$

where $X$ is the length of the directional signals, and $R_{11}\left(\tau_{x^{\prime}}\right)$ is the autocorrelation of the $g_{1}\left(x^{\prime}\right)$ signal. The autocorrelation function has a global maximum at zero and finding the maximum of the cross-correlation function at $\hat{t}_{x_{\text {max }}^{\prime}}$, the velocity estimate can be found by.

$$
\hat{v}_{x^{\prime}}=\frac{\hat{\tau}_{x_{\max }^{\prime}}}{T_{p r f}}
$$

since $\tau_{x_{\max }^{\prime}}$ is the shift in spatial position of the scatterers over the time interval $T_{p r f}$. Note that this velocity has the correct magnitude, if the directional signals have been focused along the flow direction: Note also that for a laminar flow there should be a smaller decorrelation between the two correlated signals than for a traditional system, since the signal decorrelation from different scatterer velocities are avoided.

\section{Focusing Directional Signals}

The purpose of traditional beamformers is to align all received signals from the individual elements so that they add up in phase. This is done by delaying signals that have a shorter distance to propagate than ones with a longer distance. The delay is, thus, calculated from the difference in geometric distance from one element to a reference point on the transducer. A simpler but equivalent method that can be used in a digital system is to consider the distance from the element to the point in the image to beamform. The distance divided by the speed of sound gives the time to select the sample in the signal received from the transducer element. Focusing is then achieved by adding the samples from all the elements, and this is the focusing method used in this paper.

The main challenge in this approach is to obtain the directional signals. This is done by focusing the received signals from the individual elements on the different points $\vec{r}_{x^{\prime}}$ in the directional signals. Assuming the speed of sound to be constant, the time from the scattering of the ultrasound to the reception by the transducer element is given by

$$
t_{r}=\frac{\left|\vec{r}_{e}(j)-\vec{r}_{x^{\prime}}\right|}{c}
$$

where $\vec{r}_{e}(j)$ is the position of element $j$ of the receiving transducer. The reception time is, thus, uniquely defined. The time between emission of the ultrasound pulse and the sound impinging on the point at $\vec{r}_{x^{\prime}}$ cannot be uniquely determined, since a large part of the transducer aperture is used for generating the emitted field. Several suggestions can be made. In a previous paper Jensen and Lacasa [10] suggested using

$$
t_{e}=\frac{\left|\vec{r}_{e}(j)-\vec{r}_{x^{\prime}}\right|}{c},
$$

which is the same as for the received signal. This assumes a very weakly or nonfocused field. Here, the approximate time

$$
t_{e}=\frac{\left|z^{\prime}-z_{e}^{\prime}(j)\right|}{c}
$$

will be used, where $z_{e}^{\prime}(j)$ is the $z^{\prime}$-coordinate of the receiving transducer element $j$. This is the distance from the transducer aperture to the directional line, when it is assumed that the transducer aperture is flat. Focusing on the points on the directional line is then done by

$$
g_{i}\left(x^{\prime}, z^{\prime}\right)=\sum_{j=1}^{N_{r}} g_{r}\left(j, \frac{\left|\vec{r}_{e}(j)-\vec{r}_{x^{\prime}}\right|+\left|z^{\prime}-z_{e}^{\prime}(j)\right|}{c}\right),
$$

where $g_{r}(j, t)$ is the received time signal from element $j$, $N_{r}$ is the number of receiving elements, and $t$ is time since pulse emission. This gives the directional signals as a function of depth for emission number $i$. The signals for the same depth are then cross-correlated as a function of $x^{\prime}$, and the velocity is obtained from (5) as described above. 


\section{Digital Implementation}

The bearnformation can only be made in a digital ultrasound system, and the directional signals are therefore discrete and given by $g_{i}(l)$, where the directional sampling interval is $d x^{\prime}$ and the discrete index $l$. The sample index to be used in (9) will often not be an integer, and some interpolation between two neighboring samples is needed. This can be done with a linear interpolation or by some higher order interpolation method.

The cross-correlation $R_{12}(k)$ is discrete, and the maximum found $k_{s}$ is discrete. The quantization into sampling intervals often gives a too coarse resolution in the velocity estimates, and a more precise estimate can be found by making an interpolation around the maximum point by employing [14];

$$
\begin{aligned}
& k_{i n t}= \\
& k_{s}-\frac{\hat{R}_{12}\left(k_{s}+1\right)-\hat{R}_{12}\left(k_{s}-1\right)}{2\left(\hat{R}_{12}\left(k_{s}+1\right)-2 \hat{R}_{12}\left(k_{s}\right)+\hat{R}_{12}\left(k_{s}-1\right)\right)},
\end{aligned}
$$

and the velocity is then found from

$$
\hat{v}_{x^{\prime}}=\frac{k_{i n t} d x^{\prime}}{T_{p r f}} .
$$

The cross-correlation can be improved by averaging over several estimates of $R_{12}$, since the velocity of the scatterers can be considered constant for several pulses. A number of pulse-echo lines can, thus, be used in the estimation.

The discrete cross-correlation function estimates can also yield erroneous peaks, when a limited amount of data is used, and noise is present in the data [15]. The search range in the cross-correlation function should therefore be limited to avoid these peaks. In all estimations the search range has been limited to a maximum velocity of $1 \mathrm{~m} / \mathrm{s}$, and the interpolation interval calculated by (10) has been limited to a correction within one sampling interval.

The maximum velocity that theoretically can be estimated is

$$
\hat{v}_{\max }=\frac{\max \{X\}}{T_{p r f}},
$$

where $\max \{X\}$ is the largest spatial lag in the autocorrelation function. In practice, the value is much lower, since the uncertainty in $\hat{R}_{12}$ increases with increasing lag, and it is influenced by the SNR. The maximum velocity is, thus, intrinsically linked to the actual measurement situation.

\section{E. Stationary Echo Canceling}

Stationary echo canceling can be done on the directional signals using the traditional filters. The velocity for a stationary signal is zero so that $g_{2}\left(x^{\prime}\right)=g_{1}\left(x^{\prime}-v_{x^{\prime}} T_{p r f}\right)=$ $g_{1}\left(x^{\prime}\right)$. This signal can, thus, be removed by subtracting the stationary component out. The stationary signal often overlies the blood signal, and it is, thus, necessary to isolate the stationary component. This can either be done using a simple subtraction filter or more advanced methods can be employed. In this paper, the stationary component is found from the mean of all the signals as

$$
g_{m}\left(x^{\prime}\right)=\frac{1}{N_{e}} \sum_{i=1}^{N_{e}} g_{i}\left(x^{\prime}\right),
$$

where $N_{e}$ is the number of directional signals acquired. This mean signal is then subtracted from all the directional signals

$$
g_{i, e c}\left(x^{\prime}\right)=g_{i}\left(x^{\prime}\right)-g_{m}\left(x^{\prime}\right),
$$

and this signal is used for calculating the cross-correlation function.

\section{F. Discrimination}

For a stationary signal there will be no shift between the signals so that $g_{2}\left(x^{\prime}\right)=g_{1}\left(x^{\prime}\right)$, and there is therefore no difference between the signals. The stationary echo canceling then removes all signals, and only noise is left, from which the velocity cannot be estimated. An indication of the validity of the estimate should, thus, be calculated. This can be found from the ratio between the energy of the signal before and after echo canceling given as

$$
R_{e}=\frac{\sum_{i=1}^{N_{e}} \sum_{l=1^{\dagger}}^{N_{x}} g_{i, e c}^{2}(l)}{\sum_{i=1}^{N_{e}} \sum_{l=1}^{N_{x}} g_{i}^{2}(l)}
$$

where $N_{x}$ is the number of samples in the directional signal. If this ratio is below a prescribed limit, the resulting velocity estimate is set to zero, since the echo-canceled signals are mostly noise, and the directional signals are highly correlated. The rejection ratio will depend on the noise in the system and on the angle between the emitted beam and the directional signal. The ultrasound field in general has a larger lateral than axial extent, and for angles different from $90^{\circ}$ there will be some influence from scatterers outside the current position for velocity estimation. The rejection ratio must then be higher than for $90^{\circ}$, where it is mainly noise that dominates after echo canceling. If the signals are very noisy, the rejection ratio should also be increased.

In this paper a fixed rejection ratio of 0.02 was selected for $90^{\circ}$ and 0.2 for all other angles. A more advanced approach could also derive the rejection value based on the signal-to-noise level, the magnitude of the stationary component, etc.

\section{G. Determination of Beam-To-Flow Angle}

The approach relies on knowledge of the angle between the flow vector and the direction of the emitted ultrasound beam. The current approach is to use the traditional Bmode image for this determination, as is done in a conventional spectral estimation system for correcting the estimated velocity values. No unique direction can be found 
for nonlaminar flow, and the standard deviation and bias will increase, as is also the case in traditional velocity estimation.

Ideally, an automatic approach for determining the angle should be made, and this is the topic of further studies. It should, however, be noticed that the emission of the ultrasound is independent of the angle. All angle-dependent processing is done on the received and sampled signals. Any angle can therefore be chosen after reception of the signals, and the correct velocity magnitude can always be found provided the correct angle is used.

\section{Simulations}

The performance of the directional velocity estimation has been investigated through a number of simulations. The Field II program [16], [17] has been used for all simulations. It uses spatial impulse responses to describe the ultrasound field and can be used for all transducer geometries, focusing and apodization schemes, and excitations. The received signals on the individual transducer elements are found from the summation of responses from a random collection of point scatterers. The simulation is performed for every pulse emission, and the point scatterers are then propagated between pulse.emissions according to the spatial velocity field. The flow is assumed laminar and parabolic with a velocity distribution of

$$
v(r)=v_{0}\left(1-\left(\frac{r}{R}\right)^{2}\right),
$$

where $r$ is the radial position in the vessel, $v_{0}$ is the peak velocity at the center, and $R$ is vessel radius. The propagation of the position $\vec{r}(\mathrm{t})$ of the individual scatterers is given by

$$
\vec{r}\left(t+T_{p r f}\right)=\vec{r}(t)+\vec{v}(\vec{r}(t)) T_{p r f},
$$

where $\vec{r}(t)$ is the position of the scatterer at time $t$. This propagation is also shown in Fig. 1.

The basic simulation parameters are shown in Table I. A standard 7-MHz linear array probe with a pitch slightly below $\lambda$ is used in the simulation with 128 active elements and a Hanning apodization on the elements in transmit. The transducer excitation was a single-cycle sinusoid at $7 \mathrm{MHz}$, and the impulse responses in transmit and receive were Hanning-weighted single-cycle sinusoids at $7 \mathrm{MHz}$. The emitted field is, thus, generated as for a conventional high-end ultrasound scanner. Linear array scanning can be attained by using a 256-element array and then multiplexing between the elements. In the simulation, data from a single image line are, however, investigated.

The flow in the vessel is parabolic. with a peak velocity of $v_{0}=0.5 \mathrm{~m} / \mathrm{s}$ and a radius of $10 \mathrm{~mm}$. The vessel center is placed $40 \mathrm{~mm}$ from the front face of the transducer and contains 250,000 point scatterers. The simulation of the parabolic flow has been repeated for 200 pulse-echo lines for all the different situations giving a total of 2,000 pulseecho lines with 128 received elements signals in each. The
TABLE I

Standard Parameters for Transducer and Parabolic Flow Simulation.

\begin{tabular}{lcc}
\hline Transducer center frequency & $f_{0}$ & $7 \mathrm{MHz}$ \\
Speed of sound & $c$ & $1540 \mathrm{~m} / \mathrm{s}$ \\
Wavelength & $\lambda=c / f_{0}$ & $0.22 \mathrm{~mm}$ \\
Pitch of transducer element & $w$ & $0.208 \mathrm{~mm}$ \\
Height of transducer element & $h_{e}$ & $4.5 \mathrm{~mm}$ \\
Kerf & $k_{e}$ & $0.035 \mathrm{~mm}$ \\
Number of active elements & $N$ & 128 \\
Elevation focus & $R_{e}$ & $25 \mathrm{~mm}$ \\
\hline RF lines for estimation & $N_{e}$ & 8 \\
Sampling frequency & $f_{s}$ & $100 \mathrm{MHz}$ \\
Pulse repetition frequency & $f_{p r f}$ & $5 \mathrm{kHz}$ \\
Distance between estimates & $d z$ & $0.5 \mathrm{~mm}$ \\
Sampling interval for lines & $d x=\lambda / 10$ & $0.022 \mathrm{~mm}$ \\
Correlation interval & $-10 \lambda: 10 \lambda$ & $-2.2: 2.2 \mathrm{~mm}$ \\
\hline Radius of vessel & $R$ & $10 \mathrm{~mm}$ \\
Distance to vessel center & $Z_{v e s}$ & $40 \mathrm{~mm}$ \\
Peak velocity in flow & $v_{0}$ & $0.5 \mathrm{~m} / \mathrm{s}$ \\
\hline
\end{tabular}

simulation was done on more than 40 Linux PCs ranging from a $600-\mathrm{MHz}$ Pentium III to a $2-\mathrm{GHz}$ Pentium IV using Matlab 6.1 and Field II version 3.1. One pulse emission simulation took between 1 and 3 hours on the PCs corresponding to a combined simulation time of 250 days.

Subsequently, the data were processed using Matlab for beamformation and velocity estimation. A linear interpolation between sample values was performed, when evaluating (9) to focus the beam along the flow lines. More advanced interpolation methods can be used, but this is probably of limited value, when such a high ratio between transducer and sampling frequency is used.

\section{RESULTS}

Fig. 3 shows typical signals for the velocity estimation for two consecutive emissions. The top graph shows two directional signals as a function of $x^{\prime}$ at one given depth for a parabolic velocity profile. The displacement in distance is clearly seen, and this is reflected in the cross-correlation function shown below. The lag axis on the bottom graph has been converted to velocity through (5), and the peak at $v=0.26 \mathrm{~m} / \mathrm{s}$ coincides with the correct velocity.

A typical example for a full simulation run can be seen in Fig. 4.

Typical profiles obtained at a beam-to-flow angle $\theta$ of $45^{\circ}$ are shown in Fig. 4. The mean bias over the profile is $-0.54 \%$, and the standard deviation is $1.6 \%$ relative to the true profile. Results from using the conventional crosscorrelation approach is shown in Fig. 5. The standard simulation parameters shown in Table I have been used, and no noise was added to the data. The top graph shows the individual estimated profiles, and the lower graph is the mean of the estimates \pm 3 standard deviations. For all simulation results shown on the following pages, the bias of the estimates compared to the true profile has been calculated 

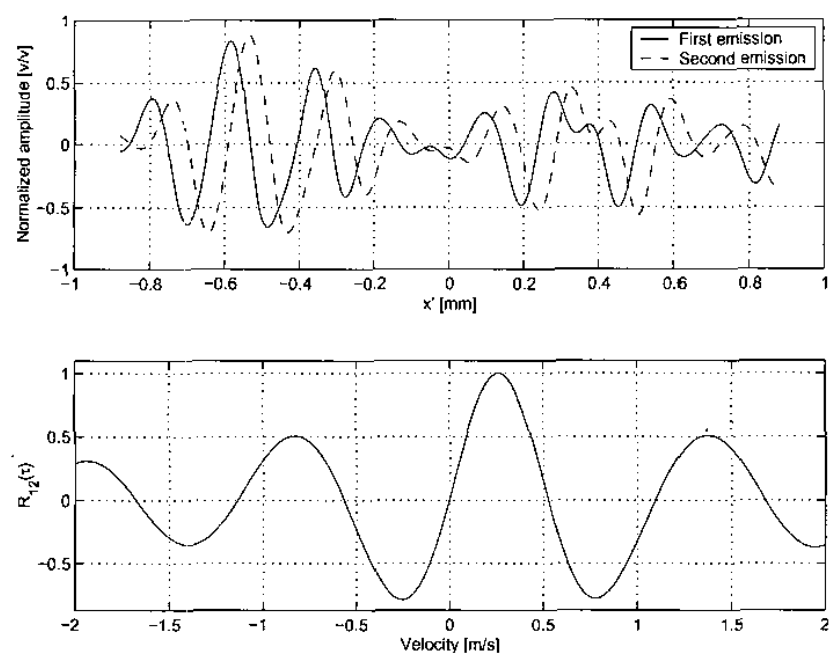

Fig. 3. Example of two directional signals (top graph) as a function of $x^{\prime}$. Corresponding cross-correlation function is shown in the bottom graph.
Parabolic profile at 45 deg., 8 pulse echo lines, focus at $40 \mathrm{~mm}$

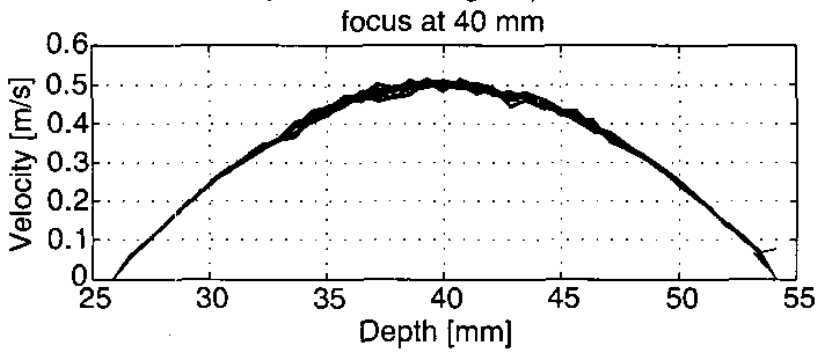

Mean $+/ 3$ std. for parabolic profile at 45 deg., 8 pulse echo lines, focus at $40 \mathrm{~mm}$

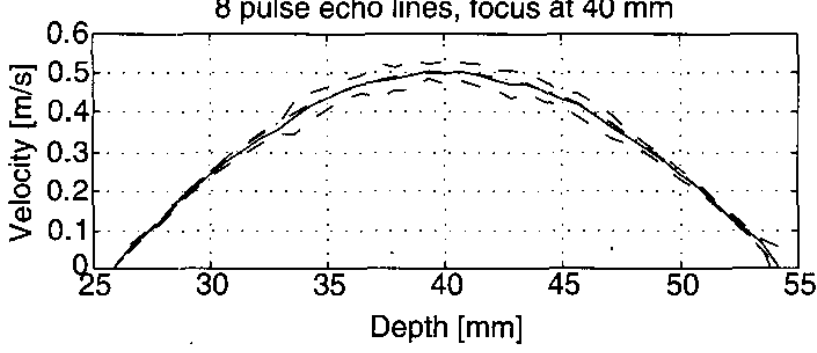

Fig. 4. Typical estimated velocity profiles at a beam to flow angle of $45^{\circ}$, when using the new approach. The top graph shows the individual profiles, and the bottom graph shows the mean (solid line) of the profiles \pm 3 standard deviations (dashed lines). The mean bias over the profile is $-0.54 \%$, and the standard deviation is $1.6 \%$ relative to the true profile.
Parabolic profile at 45 deg., 8 pulse echo lines, focus at $40 \mathrm{~mm}$

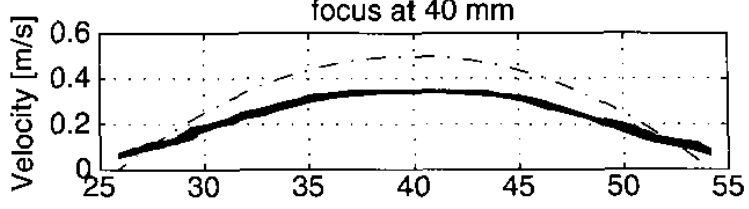

Mean $+/ 3 \mathrm{std}$. for parabolic profile at $45 \mathrm{deg}$. 8 pulse echo lines, focus at $40 \mathrm{~mm}$

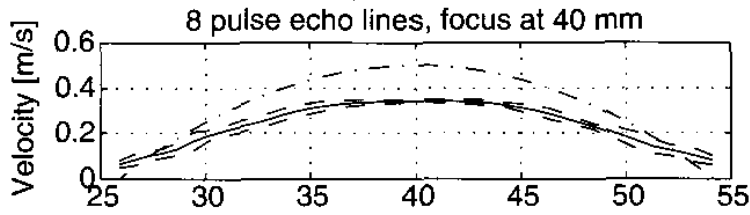

Mean $+/ 3$ std. for compensated parabolic profile at 45 deg.,

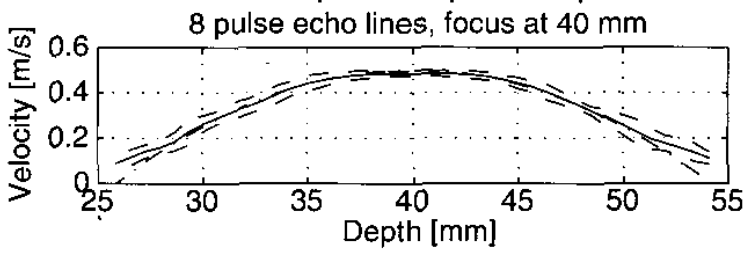

Fig. 5. Typical estimated velocity profiles at a beam to flow angle of $45^{\circ}$, when using the conventional cross-correlation approach. The top graph shows the individual profiles, and the middle graph shows the mean of the profiles \pm 3 standard deviations. The lower graph is when the velocity estimates have been compensated for the angle. Here, the mean bias over the profile is $2.5 \%$, and the standard deviation is $2.1 \%$. The dashed-dotted line is the correct velocity profile.

as the mean deviation over the profile relative to the peak velocity $v_{0}=0.5 \mathrm{~m} / \mathrm{s}$. The relative standard deviation in percent has also been found averaged over the profile compared to the peak velocity. The two top graphs show when no compensation for the angle has been made in (1). The bias is here $-17.2 \%$. Compensating for the angle gives the figure in the bottom. The mean bias over the profile is now reduced to $2.5 \%$, and the standard deviation is $2.1 \%$. The new method is, thus, an improvement over conventional flow imaging, since both bias and standard deviation are reduced. This is due to the smaller decorrelation between the lines, since the beams follow the scatterers. There is, thus, not a different velocity in different parts of the signal.

The new approach can also be used for finding the velocity transverse to the ultrasound beam, in which conventional approaches fail in estimating the velocity. This is demonstrated in Fig. 6, where the profiles for a fully transverse flow have been estimated from 8 pulse-echo signals using the new approach with stationary echo canceling. The mean bias over the profile is $-2.6 \%$, and the standard deviation is $6.6 \%$ demonstrating that the transverse flows can be found. A conventional system would here yield a velocity of zero.

\section{A. Parameter Variations}

Results from variations of the simulation parameters are shown in this section. Several parameters have been 
Parabolic profile at $90 \mathrm{deg} ., 8$ pulse echo lines, focus at $80 \mathrm{~mm}$

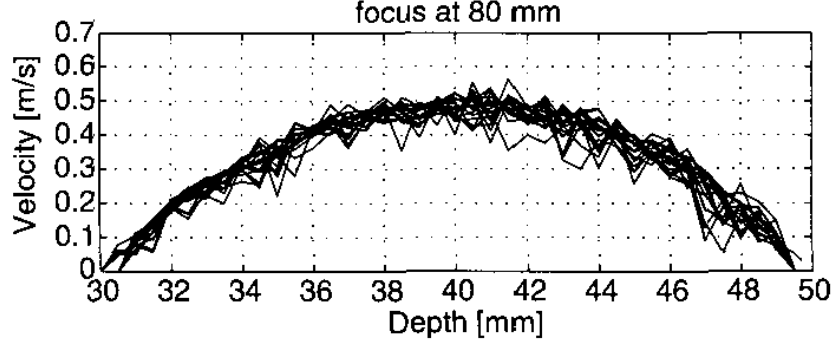

Mean $+/ 3$ std. for parabolic profile at $90 \mathrm{deg}$., 8 pulse echo lines, focus at $80 \mathrm{~mm}$

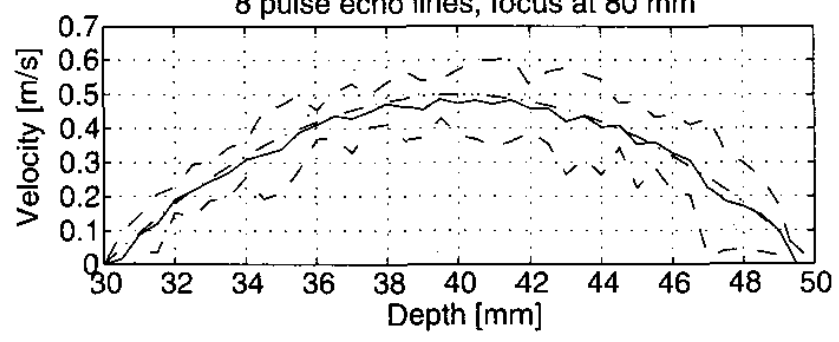

Fig. 6. Typical estimated velocity profles at $90^{\circ}$ using the new approach. The top graph shows the individual profiles, and the bottom graph shows the mean of the profiles \pm 3 standard deviations. The mean bias over the profile is $-2.6 \%$ and the standard deviation is $6.6 \%$.

varied in order to show the influence on performance of the method and its robustness.

Fig. 7 shows the performance, when the pulse repetition frequency is varied. The top four graphs show the bias of the results compared to the true parabolic velocity profile, and the lower four graphs show the standard deviation averaged over the profile. The individual graphs show the results for different beam-to-flow angles, where $90^{\circ}$ corresponds to a transverse flow to the ultrasound beam. The bias and standard deviation are in general larger for the transverse flow than for other angles, and this is reflected in the choice of axis, which covers a larger range in this situation. The individual graphs contain several lines, one for each simulation of a transmit focus. The transmit focus has been set at the center of the vessel and at distances further away.

For a focus at $40 \mathrm{~mm}$ in the vessel, both bias and standard deviation increases, when $f_{p r f}$ is decreased, and it should not be below $2000 \mathrm{~Hz}$ to give reliable estimates. For all other emission foci and all angles, both the bias and standard deviation are decreased for decreased $f_{p r f}$. This indicates that a large shift between the signals is beneficial, as the signals only decorrelate slowly, when using the new approach for a laminar acceleration free flow. For a very low $f_{p r f}$, the standard deviation will rise again, since the data get uncorrelated and the velocity does not stay constant.

Fig. 8 shows the results for a variation from the correct beam-to-flow angle. The bias is, as expected, severely affected by an error in angle. For nontransverse flow the bias follows the normal projection of the flow, whereas it is significantly different for the transverse flow. An angle error is in this case not related to the projection of the flow onto the estimation direction, but it does increase with angle error and has a minimum for the correct angle. The standard deviation is affected in the same way and also increases significantly with the angle error for a transverse flow. It is less affected for the nontransverse estimates, but a minimum is attained for the correct angle.

Fig. 9 shows the results for a variation in the sampling interval for the directional signals. The $x$-axis on the figure shows the number $N_{d}$, which $\lambda$ is divided with to give the sampling interval $\left(d x=\lambda / N_{d}\right)$, when calculating the beams. The selection of interval has a negligible influence for $90^{\circ}$ for all values of $N_{d}$. For all other angles there is a decrease in both bias and standard deviation, when the sampling interval is larger than $\lambda / 5$. After this both stay roughly constant.

The results for different numbers of directional signals used in the estimation is shown in 'Fig. 10. The crosscorrelation is calculated for each set of lines, and then these correlation functions are added. The velocity is then found from the averaged correlation function. The bias of the estimates in general decreases, when the number of lines is increased. The decrease is rapid from 4 to 10 lines, where the decrease levels out. The decrease is influenced by the stationary echo canceling, which is very sensitive for few lines. At $90^{\circ}$ a large bias is seen for a focus at $40 \mathrm{~mm}$, and a consistent underestimation of the velocity can be seen for focusing at other depths. For other angles, the bias is in general lower, when 8 or more directional signals are used. The standard deviation in general decreases with an increasing number of signals for all angles.

Results for variation in the range for the correlation are shown in Fig. 11. The range is expressed as a factor times the wavelength $\lambda$ of the ultrasound. The range has a large influence on both bias and standard deviation. Increasing the range from $4 \lambda$ to $10 \lambda$ significantly decreases both bias and standard deviation for all angles, if the transmit focus is above $40 \mathrm{~mm}$. For a focus at $40 \mathrm{~mm}$, the bias increases slightly beyond $5 \lambda$, since data outside the main beam is used for estimation. The standard deviation in general attains the final value after a range of $5 \lambda$ with a slight decrease until $10 \lambda$ at $90^{\circ}$.

Gaussian random noise has been added to the RF signals used for the results in Fig. 12. The SNR is determined by

$$
\mathrm{SNR}_{d B}=10 \log _{10} \frac{E\left\{g_{r}^{2}(t)\right\}}{E\left\{n^{2}(t)\right\}},
$$

where $g_{r}(t)$ is the received element signal, and $n(t)$ is the noise. A ratio from 0 to $40 \mathrm{~dB}$ has been used. The bias is in general not very affected by the noise. The standard deviation decreases with an increasing SNR until $20 \mathrm{~dB}$ for $90^{\circ}$ and until $10 \mathrm{~dB}$ for other angles, where the final performance is reached.

No apodization is applied during receive focusing of the flow beams. It is possible to apply such an apodization, 

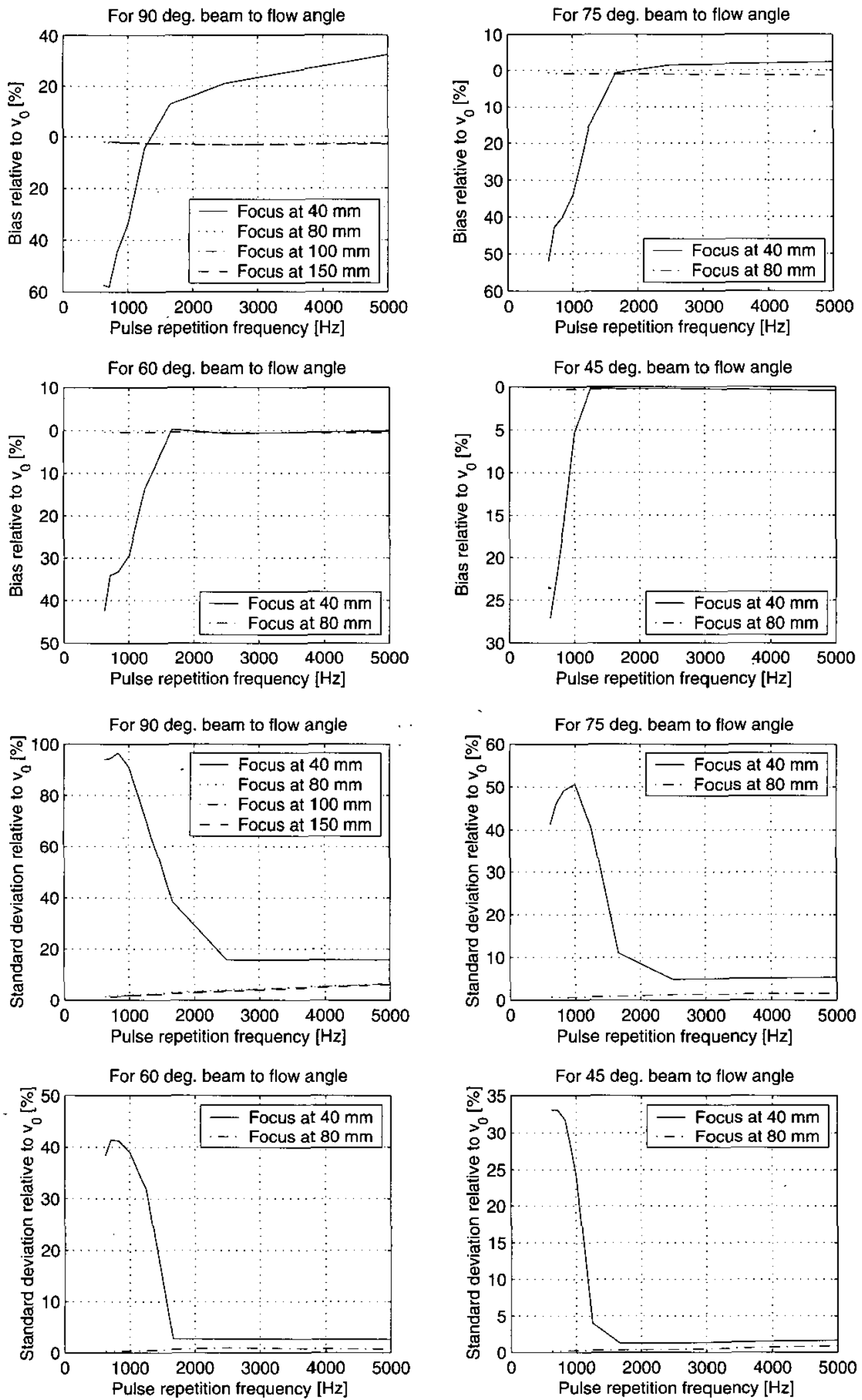

Fig. 7. Variation in bias (top) and standard deviation (bottom) for a change pulse repetition frequency. 

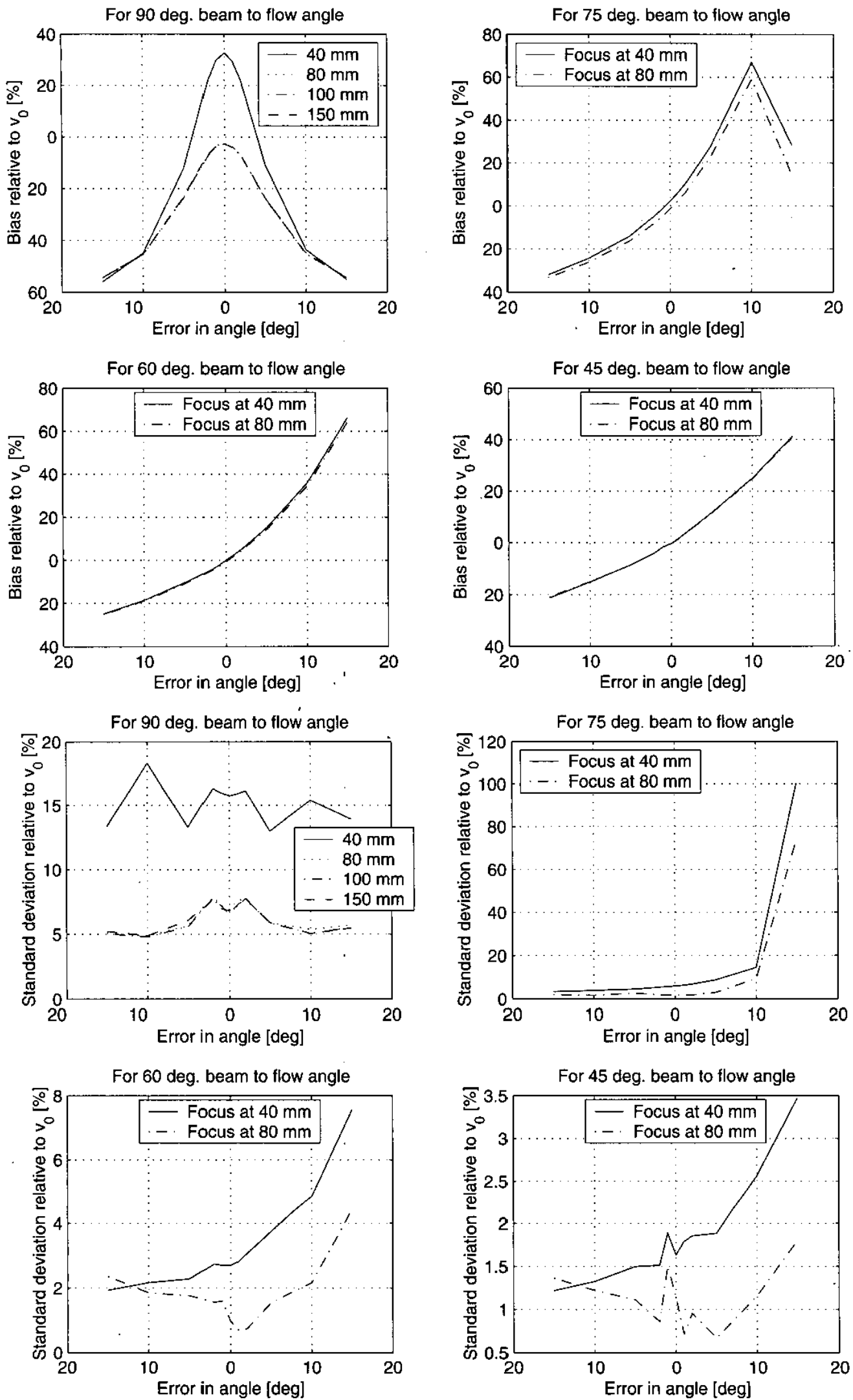

Fig. 8. Variation in angle. 

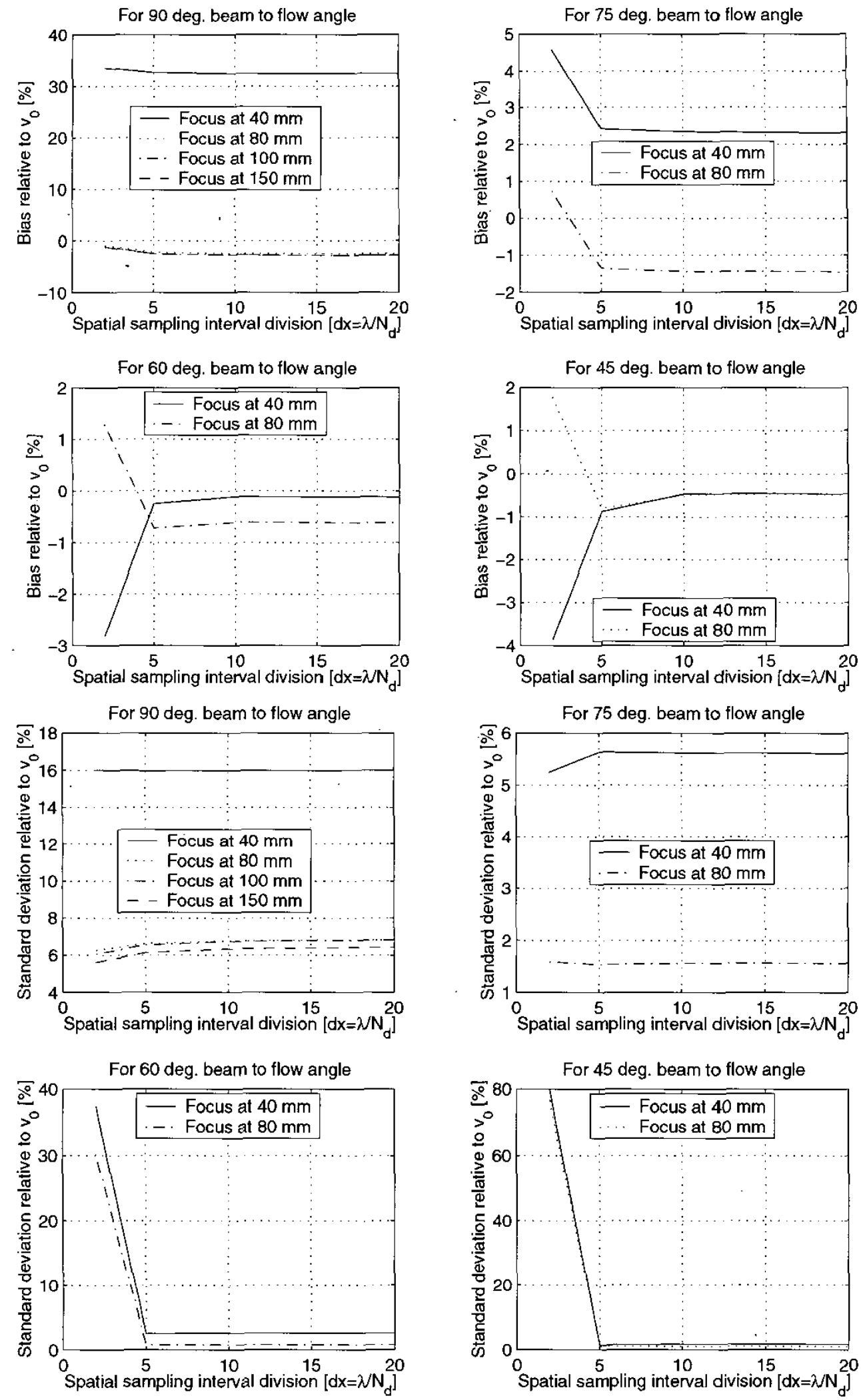

Fig. 9. Variation in sampling interval. The $x$-axis shows the number $N_{d}$ that $\lambda$ is divided with to give the sampling interval $\left(d x=\lambda / N_{d}\right)$. 

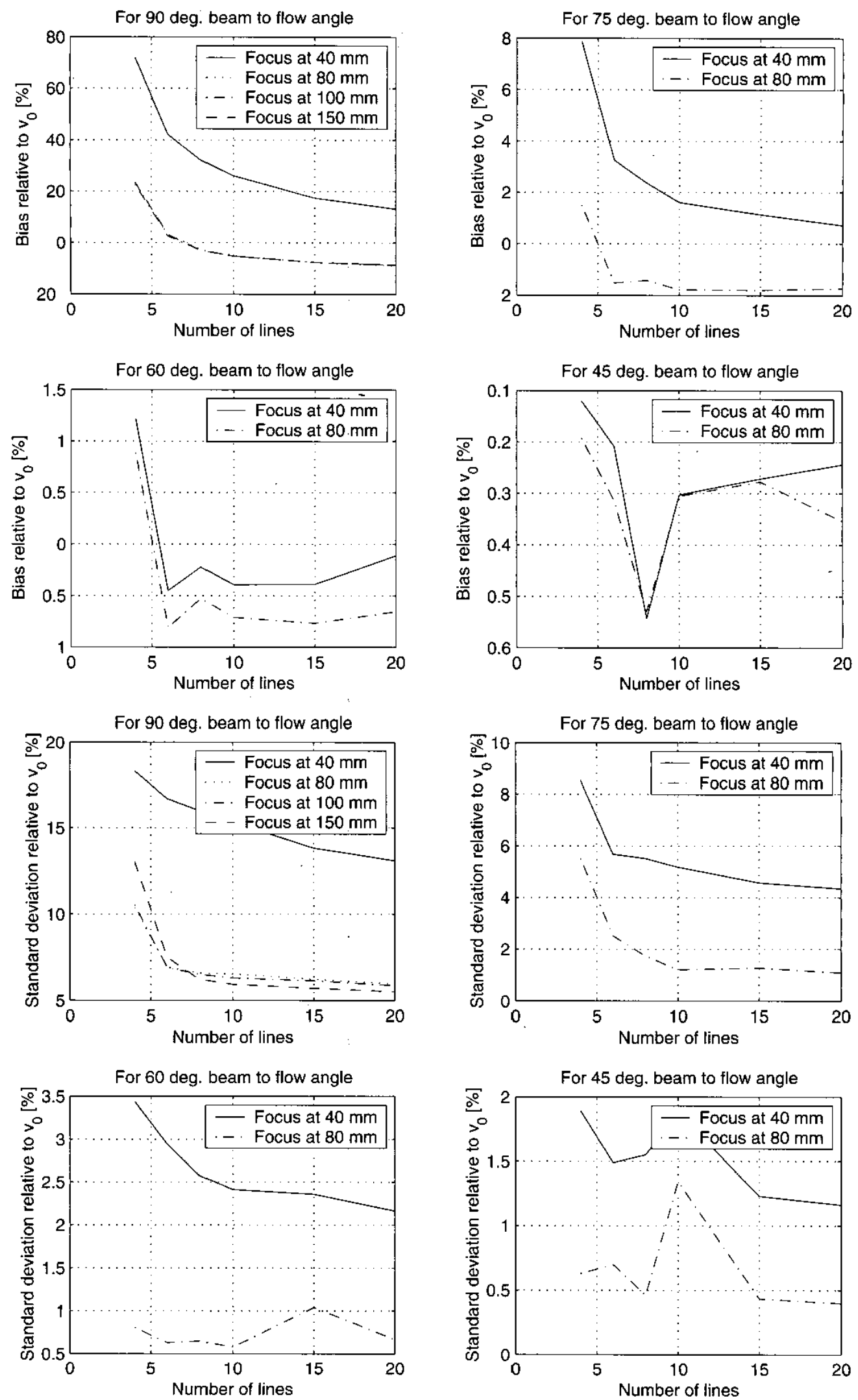

Fig. 10. Variation in number of pulse-echo lines. 

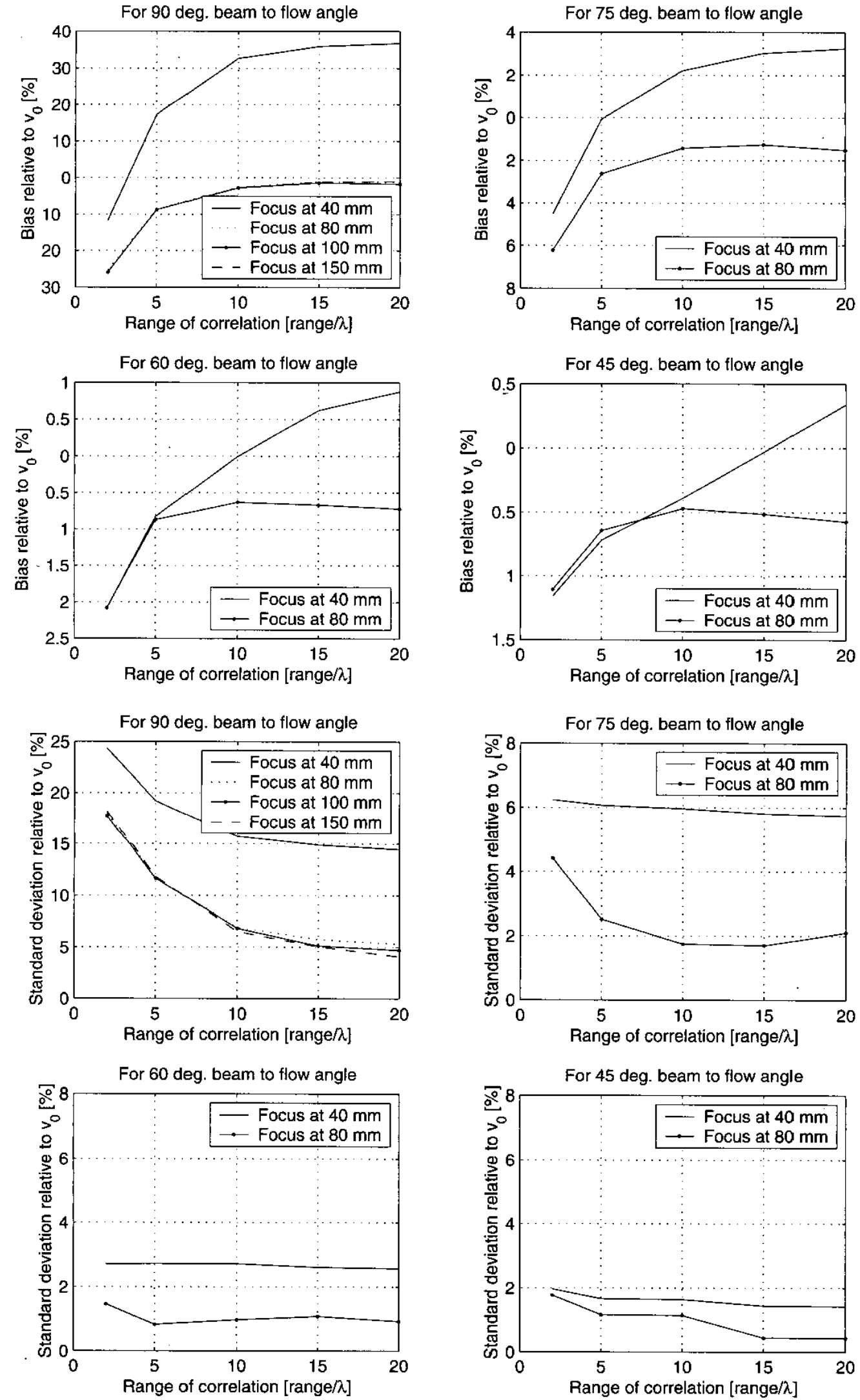

Fig. 11. Variation in correlation interval that the directional signals are calculated over expressed in wavelengths $\lambda$. 

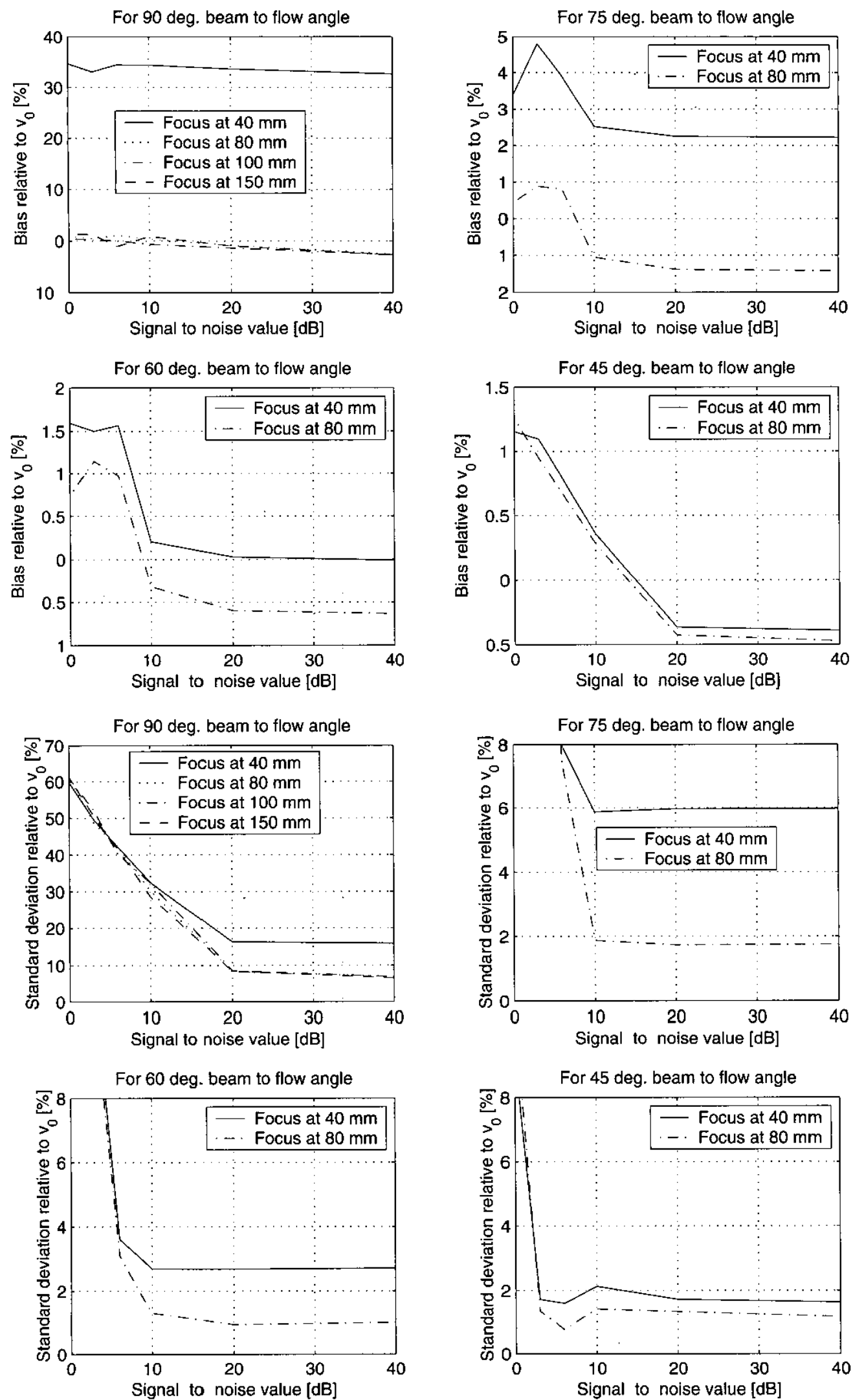

Fig. 12. Variation in bias and standard deviation as a function of SNR. 

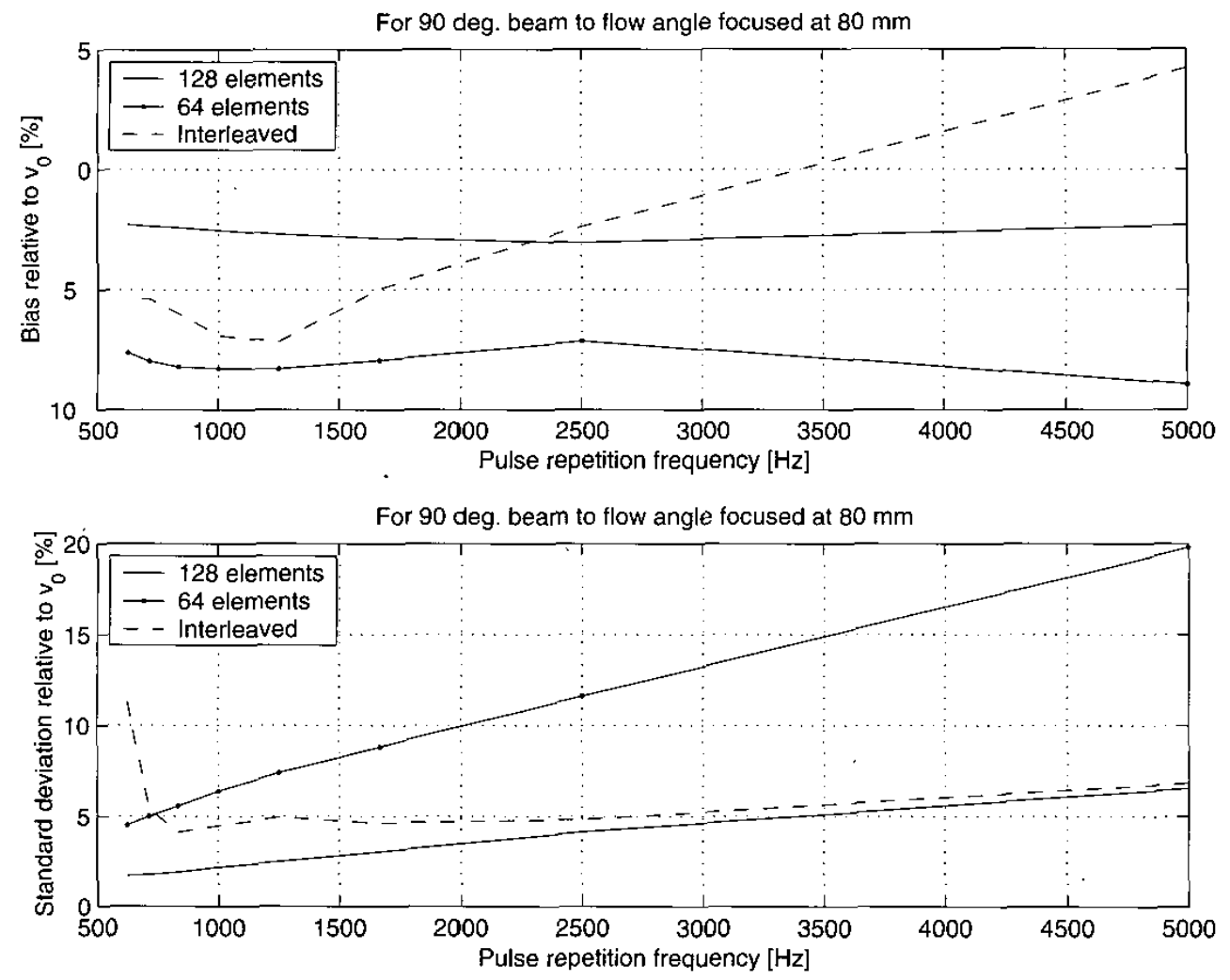

Fig. 13. Performance for different pulse repetition frequencies when using $64(-)$ and $128(-.-)$ active elements. The dashed line is when making interleaved sampling of even and odd elements.

TABLE II

Bias and Standard Deviation When Using No Apodization (Rect.) During Beam Formation and a Hanning apodization (HANN).

\begin{tabular}{crrrrc}
\hline $\begin{array}{c}\text { Angle } \\
\text { deg. }\end{array}$ & $\begin{array}{r}\text { Emit } \\
\text { focus } \\
\text { mm }\end{array}$ & $\begin{array}{r}\text { Bias } \\
\text { rect. } \\
\%\end{array}$ & $\begin{array}{r}\text { Bias } \\
\text { Hann } \\
\%\end{array}$ & $\begin{array}{r}\text { Std. } \\
\text { rect. } \\
\%\end{array}$ & $\begin{array}{c}\text { Std. } \\
\text { Hann } \\
\%\end{array}$ \\
\hline 90 & 40 & 32.6 & 47.3 & 15.8 & 39.2 \\
90 & 80 & -2.6 & -11.5 & 6.9 & 17.3 \\
90 & 100 & -2.7 & -11.5 & 6.8 & 15.8 \\
90 & 150 & -2.7 & -11.2 & 6.5 & 15.3 \\
\hline 75 & 40 & 2.2 & 2.3 & 6.0 & 7.6 \\
75 & 80 & -1.4 & -1.6 & 1.7 & 1.8 \\
\hline 60 & 40 & -0.01 & -0.18 & 2.7 & 2.9 \\
60 & 80 & -0.63 & -0.72 & 0.9 & 0.95 \\
\hline 45 & 40 & -0.39 & -0.52 & 1.63 & 1.46 \\
45 & 80 & -0.47 & -0.57 & 1.14 & 0.46 \\
\hline
\end{tabular}

and the result of this is shown in Table II. In general, both the bias and standard deviation are higher for the Hanning apodization than for the rectangular apodization, and it is, thus, not advisable to use apodization in receive.

Often scamners can only sample in 64 channels simultaneously instead of 128 . The performance of using 64 el-
TABLE III

Basic Simulation Parameters for Transducer, Flow, and Estimation in the Femoral artery.

\begin{tabular}{llc}
\hline Transducer center frequency & $f_{0}$ & $5 \mathrm{MHz}$ \\
Speed of sound & $c$ & $1540 \mathrm{~m} / \mathrm{s}$ \\
Wavelength & $\lambda=c / f_{0}$ & $0.308 \mathrm{~mm}$ \\
Width of transducer element & $w=\lambda$ & $0.308 \mathrm{~mm}$ \\
Height of transducer element & $h_{e}$ & $5 \mathrm{~mm}$ \\
Kerf & $k_{e}=\lambda / 10$ & $0.0308 \mathrm{~mm}$ \\
Number of active elements & $N$ & 128 \\
Emission focus & $F_{e}$ & $100 \mathrm{~mm}$ \\
\hline RF lines for estimation & $N_{e}$ & 8 \\
Pulse repetition frequency & $f_{p r f}$ & $5 \mathrm{kHz}$ \\
Distance between estimates & $d z$ & $0.25 \mathrm{~mm}$ \\
Sampling interval for lines & $d x=\lambda / 10$ & $0.0308 \mathrm{~mm}$ \\
Correlation interval & $-10 \lambda: 10 \lambda$ & $-3.08: 3.08 \mathrm{~mm}$ \\
\hline Radius of vessel & $R$ & $4 \mathrm{~mm}$ \\
Distance to vessel center & $Z_{v e s}$ & $60 \mathrm{~mm}$ \\
Ratio between tissue and blood & & $40 \mathrm{~dB}$ \\
\hline
\end{tabular}

ements in both transmit and receive is shown in Fig. 13 for a variation in the pulse repetition frequency. The emit focus is at $80 \mathrm{~mm}$. The top graph shows the bias and the lower the standard deviation. The solid line is when using all 128 elements. The line with dots is when using only 64 elements. It can been seen that both bias and standard 

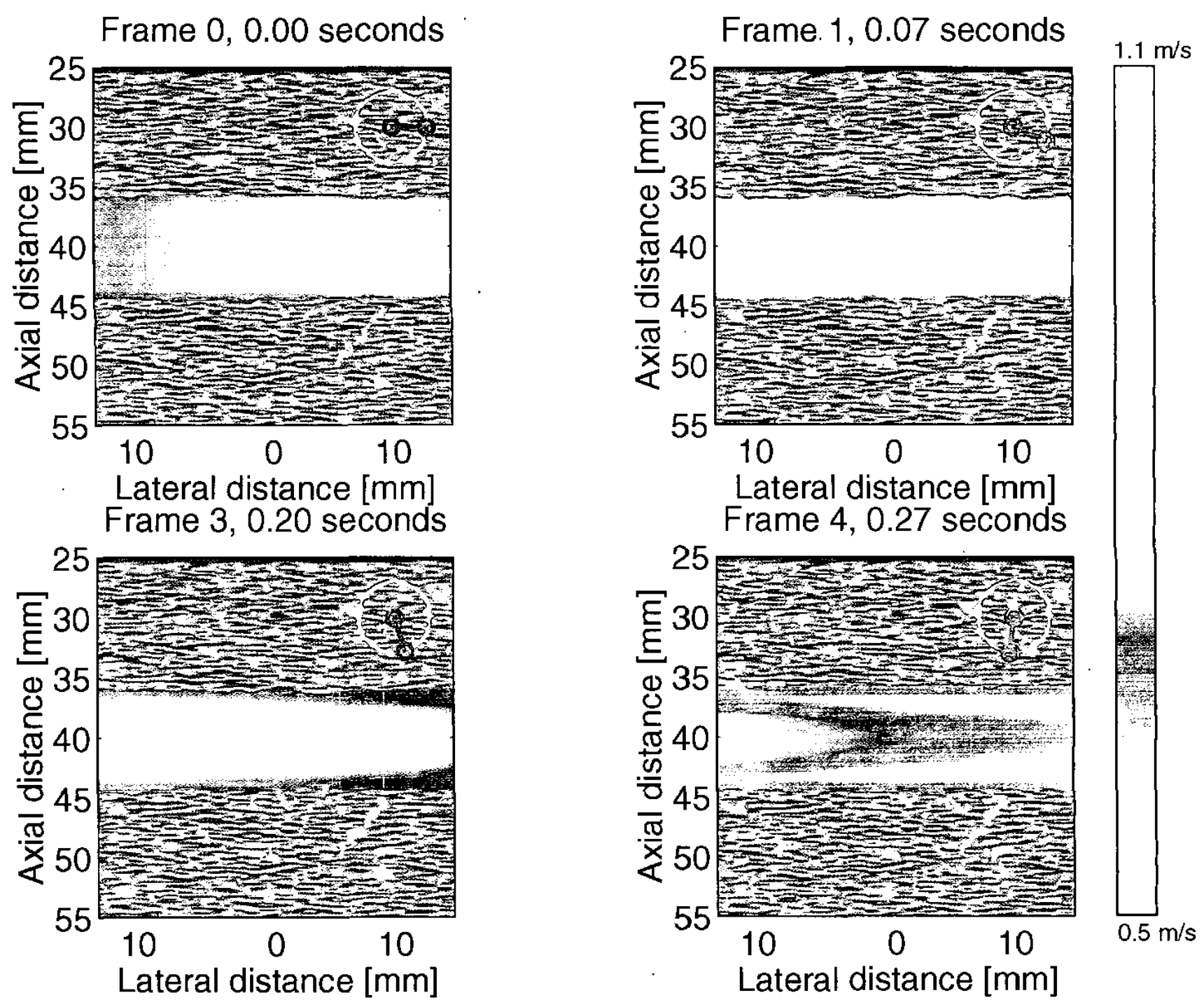

Fig. 14. Simulated color flow mapping images from the femoral artery for a purely transverse flow. A red color indicates flow towards the left and a blue color to the right. The circle and line in the top right corner indicate the phase in the cardiac cycle.

deviation deteriorate. A possible solution to this problem is to use multiplexing between the 64 even and 64 odd elements of the aperture's 128 elements. This has been done in the dashed curve. The odd elements have been measured for odd values of the signal number $i$ and even elements have been measured for even $i$. The directional signals are then beamformed for each signal, and the signal from emission $i$ is added to the signal from emission $i+1$, and these combined signals are cross-correlated. Hercby, all 128 elements are sampled and used, and the performance is improved. The bias is still worse than for 128 elements, but the standard deviation is quite close to the 128-element system.

\section{Transverse Color Flow Imaging}

Finally, a color flow mapping image with a transverse flow has been simulated. The Womersley model for pul- satile flow in the femoral artery has been used [1], [18], [19]. A realistic model of the temporal and spatial variation in the flow is thereby introduced.

The basic simulation parameters are shown in Table III. Note that a $5-\mathrm{MHz}$ transducer is used here. The parameters are the same as would be used in a modern scanner for traditional color flow mapping. Eight pulse-echo lines are used for the estimation, and stationary echo canceling is done by subtracting the mean value of the beamformed data for a given time. The resulting color flow map images are shown in Fig. 14 for the different positions in the cardiac cycle. A red color value indicates flow to the left and a blue value to the right. Notice the change of direction of the flow at $0.27 \mathrm{~s}$, which is typical for flow in the femoral artery at rest. No postprocessing has been made on the images, and the discrimination between flow and tissue is done as described in Section II-F with a rejection value of 0.00001 . 


\section{CONCLUSION}

A new method for directional velocity estimation has been introduced. The scheme can be used in traditional delay-sum beamforming with a focused transmit field and gives a satisfactory performance with standard linear array transducers. The performance is in general increased by using a longer pulse repetition time and more recciving elements. It is robust to noise and can use only 8 to 16 pulse-echo lines for finding the velocity estimate in the case of complicated temporally and spatially varying flow in the arteries.

\section{ACKNOWLEDGMENT}

This work was supported by grants 9700883 and 9700563 from the Danish Science Foundation and by BK Medical A/S, Denmark.

\section{REFERENCES}

[1] J. A. Jensen, Estimation of Blood Velocities Using Ultrasound: A Signal Processing Approach. New York: Cambridge Univ. Press, 1996.

[2] C. Kasai, K. Namekawa, A. Koyano, and R. Omoto, "Real-time two-dimensional blood flow imaging using an autocorrelation technique," IEEE Trans. Sonics Ultrason., vol. 32, no. 3, pp. $458-463,1.985$.

[3] O. Bonnefous and P. Pesqué, "Time domain formulation of puise-Doppler ultrasound and blood velocity estimation by cross correlation," Ultrason. Imag., vol. 8, no. 2, pp. 73-85, 1986.

[4] M. D. Fox, "Multiple crossed-beam ultrasound Doppler velocimetry," IEEE Trans. Sonics Ultrason., vol. 25, no. 5, pp. $281-286,1978$.

[5] G. E. Trahey, J. W. Allison, and O. T. von Ramm, "Angle independent ultrasonic detection of blood flow," IEEE Trans. Biomed. Eng., vol. 34, no. 12, pp. 965-967, 1987.

[6] P. Munk, "Estimation of the 2-D flow vector in ultrasonic imaging: A new approach," Masters thesis, Department of Information Techrology, Technical University of Denmark, 1996.

[7] J. A. Jensen and P. Munk, "A new method for estimation of velocity vectors," IEEE Trans. Ultrason., Ferroelect., Freq. Contr., vol. 45, no. 3, pp. 837-851, 1998.

[8] M. E. Anderson, "Multi-dimensional velocity estimation with ultrasound using spatial quadrature," IEEE Trans. Ultrason., Ferroelect., Freq. Contr., vol. 45 , no. 3, pp. 852-861, 1998.

[9] O. Bonnefous, "Measurement of the complete (3D) velocity vector of blood flows," in Proc. IEEE Ultrason. Symp., 1988, pp. $795-799$.

[10] J. A. Jensen and I. R. Lacasa, "Estimation of blood velocity vectors using transverse ultrasound beam focusing and cross- correlation," in Proc. IEEE Ultrason. Symp., 1999, pp. 14931497.

[11] J. A. Jensen and P. Gori, "Improved accuracy in the estimation of blood velocity vectors using matched filtering," in Proc. IEEE Ultrason. Symp., vol. 2, 2000, pp. 1525-1530.

[12] J. A. Jensen and R. Bjerngaard, "Directional velocity estimation using focusing along the flow direction: II: Experimental investigation," IEEE Trans. Ultrason., Ferroelect., Freq. Contr., vol. 50, no. 7, pp. 873-880, 2003.

[13] D. Dotti, E. Gatti, V. Svelto, A. Uggè, and P. Vidali, "Blood flow measurements by ultrasound correlation techniques," Energia Nucleare, vol. 23, no. 11, pp. 571-575, 1976.

[14] S. G. Foster, "A pulsed ultrasonic flowmeter employing time domain methods," Ph.D. dissertation, Dept. Elec. Eng., University of Illinois, Urbana, IL, 1985.

[15] J. A. Jensen, "Artifacts in velocity estimation using ultrasound and cross-correlation," Med. Biol. Eng. Comp., vol. 32, pp. 165$170,1994$.

[16] J. A. Jensen and N. B. Svendsen, "Calculation of pressure fields from arbitrarily shaped, apodized, and excited ultrasound transducers," IEEE Trans. Ultrason., Ferroelect., Freq. Contr., vol. 39, pp. $262-267$, Jul. 1992.

[17] J. A. Jensen, "Field: A program for simulating ultrasound systems," Med. Biol. Eng. Comp., in 10th Nordic-Baltic Conference on Biomedical Imaging, 1996, vol. 4, supplement 1, pp. 351-353.

[18] J. R. Womersley, "Oscillatory motion of a viscous liquid in a thin-walled elastic tube. I: The linear approximation for long waves," Phil. Mag., vol. 46, pp. 199-221, 1955.

[19] D. H. Evans, "Some aspects of the relationship between instantaneous volumetric blood flow and continuous wave Doppler ultrasound recordings I," Ultrasound Med. Biol., vol. 8, no. 6, pp. 605-609, 1982.

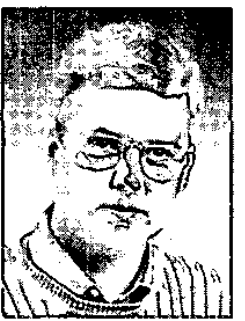

Jørgen Arendt Jensen (M'93-S'02) earned his Master of Science degree in electrical engineering in 1985 and the Ph.D. degree in 1989 , both from the Technical University of Denmark, Lyngby. He received the Dr.Techn. degree from the university in 1996 . He has published a number of papers on signal processing and medical ultrasound and the book "Estimation of Blood Velocities Using Ultrasound," Cambridge University Press in 1996. He has been a visiting scientist at Duke University, Stanford University, and the University of Illinois at Urbana-Champaign. He is currently full professor of Biomedical Signal Processing at the Technical University of Denmark at the Departinent of Information Technology and head of Center for Fast Ultrasound Imaging. He has given courses on blood velocity estimation at both Duke University and the University of Illinois and teaches biomedical signal processing and medical imaging at the Technical University of Denmark. He arranged an international summer school on advanced ultrasound imaging in 1999. 